\title{
EFFECT OF TREADMILL GAIT TRAINING ON DIPLEGIC CHILDREN SWING PHASE GAIT PARAM ETERS
}

Mariam A. Ameer $* 1$, Mohamed E. Khallaf ${ }^{2}$.

${ }^{{ }^{*}}$ Department of biomechanics, Faculty of Physical Therapy, Cairo University, Egypt.

Department of Physical Therapy, Faculty of Applied M edical Sciences, Dammam University, Saudi Arabia.

${ }^{2}$ Department of Physical Therapy for Neuromuscular Disorders and its Surgery, Faculty of Physical Therapy, Cairo University, Egypt.

\section{ABSTRACT}

Background: Swing phase of walking is considered as one of the most affected phase of gait in diplegic children. This study aimed at studying the effect of task oriented intervention on the Kinetic and kinematic parameters during swing phase of spastic diplegic children.

Materials and Methods: Twenty diplegic children were selected and distributed into two equal groups. Control group received a traditional treatment and an experimental group treated with traditional treatment and treadmill gait training. Gait analysis was done by 3-dimensional motion analysis system with 10 infrared cameras and two force platforms.

Results: Cadence, walking speed, step length, stride length, maximum knee flexion angle, maximum ankle dorsiflexion angle, maximum knee flexion moment and maximum ankle dorsiflex moment showed statistically significant improvement in both groups at Pd" 0.05. Single limb support time showed non-significant improvements in both groups at Pd" 0.05 .

Conclusion: The results indicated that the use of task oriented training in the form of treadmill gait training and the conventional physical therapy treatment improve spatiotemporal parameters, knee flexion angle and ankle dorsiflexion moment and decrease the knee flexion moment in swing phase.

KEY WORDS: Diplegic children; Treadmill; Swing phase; Gait training.

Address for correspondence: Dr.M ariam A. Ameer PT PhD., Faculty of Applied M edical Sciences, Dammam University, Saudi Arabia. Institutional Address: Al Akrabia, Al-Khober, KSA.

E-Mail: mariam_ameer7@hotmail.com OrMamahmoud@ud.edu.sa

\begin{tabular}{|c|c|c|}
\hline \multicolumn{3}{|c|}{ Access this Article online } \\
\hline \multirow{2}{*}{$\begin{array}{c}\text { Quick Response code } \\
\text { Dol: 10.16965/ijpr.2016.200 }\end{array}$} & \multicolumn{2}{|c|}{$\begin{array}{l}\text { International Journal of Physiotherapy and Research } \\
\text { ISSN } 2321-1822 \\
\text { www.ijmhr.org/ijpr.html }\end{array}$} \\
\hline & $\begin{array}{l}\text { Received: 29-11-2016 } \\
\text { Peer Review: 29-11-2016 } \\
\text { Revised: None }\end{array}$ & $\begin{array}{l}\text { Accepted: } 20-12-2016 \\
\text { Published (O): 11-02-2017 } \\
\text { Published (P): 11-02-2017 }\end{array}$ \\
\hline
\end{tabular}

\section{INTRODUCTION}

Cerebral palsy is a non-progressive disturbance in the brain functions occurring before, during, or shortly after birth [1] and it is the most common motor disability in childhood [2,3]. Cerebral palsy was one of the most common neurologic disorders among Saudi children with a prevalence rate of $23.4 / 10000$. It leads to disorders of movement and posture causing disorders of movement and posture causing activity limitations [4]. Spastic CP is the most common form of CP and fifty percent of children with spastic CP have diplegia. In spastic diplegic $\mathrm{CP}$, the lower limbs are severely impaired but the upper limbs are only mildly affected. Intelligence is usually normal with uncommon epilepsy [5].

Spastic diplegic children can walk independently. 
Gait abnormalities have been recognized as a common problem in those children especially the swing phase of gait [6]. Gait of spastic diplegic children is characterized by landing with forefoot, increase the internal rotation of the whole legs at the hip joint, limitation of lower limb joints ranges of motion, excessive hip flexion in the stance phase, and decreased foot clearance in swing phase [7,8].

Current theories of motor control and motor learning support the use of task oriented approach for addressing individuals with neurological deficits [9]. Task-oriented training is used as a rehabilitation strategy to improve motor skill, muscle strength or function $[10,11]$. It can be performed through repeated training of activity tasks as treadmill training [12]. The majority of researches on the principles that underpin effective task-oriented interventions for clients with neurological disorders has been done with persons with stroke [9]. For children with CP, task-oriented interventions using treadmill training improved lower extremity function and spatiotemporal parameters during gait. However, the differences between groups of experiments, were mostly not significant [13]. Moreover, detailed kinematic and knee flexion and ankle dorsiflexion moments during swing phase are not fully studied. In addition, a small number of studies, which is also a small sample of participants with heterogeneity of the condition. This can create wide distributions of baseline and change scores making it difficult to detect change and identify best responders and non-responders.

In this study, we selected the most common type of spastic CP with a trail to increase homogeneity of cases to study the effect of task oriented intervention on the Kinetic and kinematic parameters during swing phase of spastic diplegic children. It was hypothized that task oriented intervention in the form of treadmill training is effective in correcting swing phase Kinetic and kinematic gait parameters of spastic diplegic children.

\section{MATERIALS AND METHODS}

Twenty spastic diplegic children (12 males and 8 females) represented the sample of the study. They were recruited from King Fahd Hospital in
Al-Khober, KSA. Subjects were included in the study if their ages between five to nine years; gross motor function (GM FCS) of level I and II; mild spasticity according to the Modified Ashworth Scale (MAS). Also, they were able to walk independently for 10 meters with the absence of cognitive, auditory or visual impairments. Subjects were excluded from the study if there is history of cardiovascular disorders, neuromuscular problems other than $\mathrm{CP}$ or orthopedic surgeries as well as leg length discrepancy. Study was approved by ethical committee of Dammam University and parents signed a consent form for participation. Participants were assigned randomly into two equal groups. Randomization schedule was prepared using computer by an independent researcher who was not aware of the numeric code for study $\left(G_{1}\right)$ and control $\left(G_{2}\right)$ groups. Allocation into the groups was made by another independent researcher, who was also uninformed about the codes.

Before randomization, VICON optical motion capture system (VICON M otion Systems, Oxford, UK) used for kinetic and kinematic gait analysis. Eight cameras and 16 anatomical reflective markers were selected for the plug-in-gait model. For kinematic analysis, the markers were attached bilaterally on both lower limbs as follow: posterior superior iliac spine, anterior superior iliac spine, middle thigh, lateral axe of the knee joint, middle tibia, lateral malleolus, posterior aspect of the heel, and the base of the second toe. After system calibration and a brief demonstration about measurement procedures, child was instructed to walk with the usual speed along the walk-way of the motion analysis system without assistance. During walking, each foot was placed on the corresponding force platform for kinetic analysis. Data collection was processed using Nexus 2.2 and Polygon 3.5 software to get the XYZ (sagittal, frontal, transverse planes) coordinates. The following kinematic and kinetic parameters were obtained: cadence, walking speed, step length, stride length, single limb support time, maximum knee flexion- swing, maximum knee flexion moment- swing, and ankle dorsiflexion moment-swing.

For interventions, children in both control and 
experimental groups, received thirty minutes of traditional physical therapy, two times a week for seven consecutive weeks. The therapy session included strengthening exercises for the hip abductors, knee extensors and ankle planter flexors; and stretching exercises for the hip adductors, knee flexors, ankle planter flexors, shoulder adductors and shoulder internal rotators as well as balance and functional training.

For the study group, a protocol of treadmill gait training involving two sessions per week for a period of seven weeks was applied. The protocol employed in the present study was two 30-minute sessions per week over seven successive weeks. At the first and second times, familiarization period was considered with the treadmill. Training was gradually increased in speed. When needed, the components of the gait cycle were facilitated during training. Sixty to eighty percent maximal tolerance reached on the initial treadmill test was used for the training sessions. Walking speed was at $60 \%$ maximal speed in the first and terminal five minutes of each session and at $80 \%$ maximal speed for the other 20 minutes [14] (Figure 1).

Fig. 1: Spastic dipelgic children during treadmill gait training.

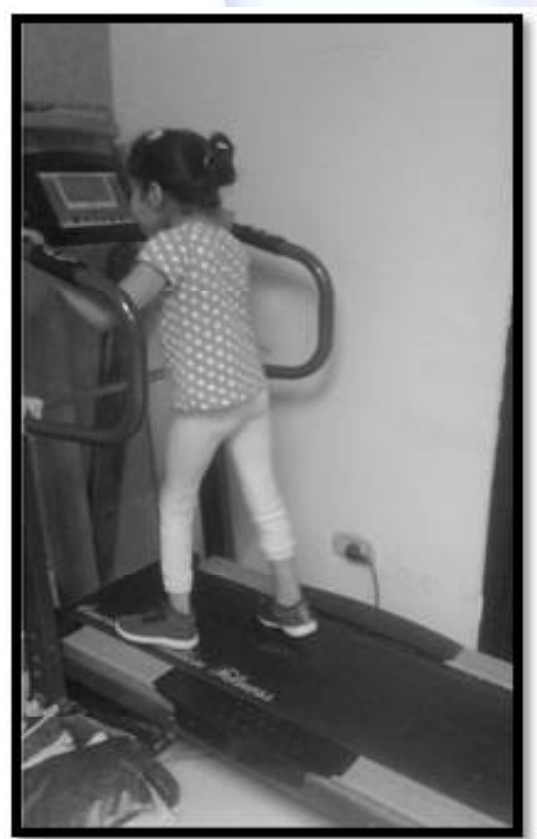

Descriptive statistics were calculated to summarize the demographic characteristics of the sample and all outcome measures at baseline (1st measurement) and post-intervention (2nd measurement) for each group. Demographic data was compared between groups by t-test $(P \leq 0.05)$. Data analyses were performed by using SPSS version 20 (SPSS Inc, Chicago, IL, USA). Independent sample t-test was employed to calculate, within group, effect of treatment programs in the study and control group at probability level less than 0.05. Between groups, effects of the applied treatment programs were compared using t-test with level of significance were set at $P \leq 0.05$.

\section{RESULT}

The characteristics of the participants are shown in Table 1. The two groups were matched for age, weight, and height ( $p=0.564,0.334$, and 0.771 respectively) as well as body mass index $(p=0.346)$. For the purpose of gait analysis, there were nonsignificant differences between the two groups in leg length $(P=0.297$ for right leg and 0.08 for left leg), knee width ( $P=0.245$ for right knee and 0.266 for left one) and ankle width $(P=0.839$ for right ankle and 0.854 for left ankle).

Table 2 and graph 1 detail the results of within and between groups comparisons. There were non-significant differences between the two groups in the base line measurements of the spatiotemporal, kinematic and kinetic parameters of gait $(P \geq 0.05)$. After interventions, significant differences have been observed in both groups with more improvements in the study group than the control group. In the study group, there were significant differences (within group comparison) between baseline measurement and follow up in cadence $(p=0.013)$, Walking speed $(p=0.000)$, Step length $(p=0.002)$, Stride length $(p=0.004)$. in the same them a significant difference was observed with Maximum knee flexion angle, Maximum ankle dorsiflexion angle, maximum Knee flexion moment and maximum ankle dorsiflexion moment $(P=0.003,0.000,0.039$ and 0.000 respectively). On the other hand, the single limb support time showed a nonsignificant difference between the baseline and follow up measures $(p=0.534)$.

In the control group, there were also significant differences between baseline and follow up measurements in cadence $(p=0.022)$, Walking speed $(p=0.049)$, Step length $(p=0.007)$, Stride 
Table 1: Demographic Data of children presented in the study.

\begin{tabular}{|c|c|c|c|c|}
\hline & & $\begin{array}{c}\text { Study group }\left(\mathbf{G}_{1}\right) \\
\text { Mean } \pm S D\end{array}$ & $\begin{array}{c}\text { Control group }\left(G_{2}\right) \\
\text { Mean } \pm S D\end{array}$ & $\mathbf{P}$ \\
\hline \multicolumn{2}{|c|}{ Age (years) } & $6.5 \pm 1.15$ & $6.8 \pm 1.36$ & 0.564 \\
\hline \multicolumn{2}{|c|}{ Height $(\mathrm{cm})$} & $111.2 \pm 7.54$ & $115.2 \pm 10.26$ & 0.334 \\
\hline \multicolumn{2}{|c|}{ Weight (Kg) } & $31.41 \pm 4.20$ & $32.07 \pm 5.67$ & 0.771 \\
\hline \multicolumn{2}{|l|}{ BMI } & $25.38 \pm 2.58$ & $24.15 \pm 3.08$ & 0.346 \\
\hline \multirow{2}{*}{$\begin{array}{l}\text { Leg length } \\
\text { (cm) }\end{array}$} & Rt & $56.15 \pm 4.31$ & $58.4 \pm 5.04$ & 0.297 \\
\hline & Lt & $55.18 \pm 2.68$ & $58.6 \pm 5.17$ & 0.080 \\
\hline \multirow{2}{*}{$\begin{array}{l}\text { Knee width } \\
\text { (cm) }\end{array}$} & Rt & $6.94 \pm .81$ & $7.40 \pm .90$ & 0.245 \\
\hline & Lt & $6.97 \pm .85$ & $7.43 \pm .94$ & 0.266 \\
\hline \multirow{2}{*}{$\begin{array}{l}\text { Ankle width } \\
\text { (cm) }\end{array}$} & $\mathbf{R t}$ & $5.35 \pm .90$ & $5.43 \pm .84$ & 0.839 \\
\hline & Lt & $5.37 \pm .84$ & $5.44 \pm .84$ & 0.854 \\
\hline
\end{tabular}

Table 2: Within and Between groups comparison of spatiotemporal, kinematic and kinetic variables before and after interventions. Data is presented as mean \pm SD. Knee flexion and Ankle dorsiflexion angles were measured in the sagittal plane.

\begin{tabular}{|c|c|c|c|c|c|c|c|c|c|c|}
\hline \multirow{3}{*}{ Variables } & \multicolumn{6}{|c|}{ Within group } & \multicolumn{4}{|c|}{ Between groups } \\
\hline & \multicolumn{3}{|c|}{ Study group ( $n=10$ ) } & \multicolumn{3}{|c|}{ Control group ( $n=10$ ) } & \multicolumn{2}{|c|}{ Before intervention } & \multicolumn{2}{|c|}{ After intervention } \\
\hline & Pre & Post & $\mathbf{P}$ & Pre & Post & $\mathbf{P}$ & $t$ & $p$ & $t$ & $p$ \\
\hline Cadence (steps/min) & $105.17 \pm 7.89$ & $115 \pm 6.60$ & $.013^{*}$ & $102.11 \pm 6.86$ & $105.99 \pm 8.09$ & $.022^{*}$ & -0.926 & 0.366 & -2.728 & $.014^{*}$ \\
\hline Walking speed(m/s) & $0.78 \pm 0.12$ & $0.92 \pm 0.15$ & $.000^{*}$ & $0.78 \pm 0.14$ & $0.77 \pm 0.13$ & $.049 *$ & 0.088 & 0.931 & -2.252 & $.037 *$ \\
\hline Step length $(m)$ & $0.35 \pm 0.05$ & $0.47 \pm 0.08$ & $.000 *$ & $0.34 \pm 0.06$ & $0.35 \pm 0.07$ & $.007 *$ & -0.74 & 0.469 & -3.622 & $.002 *$ \\
\hline Stride length $(\mathrm{m})$ & $0.70 \pm 0.11$ & $0.95 \pm 0.16$ & $.000 *$ & $0.67 \pm 0.12$ & $0.71 \pm 0.14$ & $.001 *$ & -0.726 & 0.477 & -3.5 & $.003^{*}$ \\
\hline $\begin{array}{l}\text { Single limb support time } \\
\text { (Seconds) }\end{array}$ & $0.40 \pm 0.07$ & $0.41 \pm .07$ & 0.534 & $0.43 \pm 0.07$ & $0.43 \pm 0.08$ & 0.421 & 0.646 & 0.526 & 0.661 & 0.517 \\
\hline \begin{tabular}{|c} 
Maximum knee flexion angle \\
$\left({ }^{\circ}\right)$
\end{tabular} & $56.65 \pm 3.71$ & $61.89 \pm 2.54$ & $.003^{*}$ & $55.89 \pm 4.32$ & $57.44 \pm 3.46$ & $.009 *$ & -0.424 & 0.677 & -3.281 & $.004 *$ \\
\hline $\begin{array}{c}\text { Max. Ankle dorsiflexion } \\
\text { angle }\left(^{\circ}\right) \\
\end{array}$ & $-10.79 \pm 13.93$ & $9.9 \pm 13.3$ & $.000 *$ & $-13.28 \pm 14.03$ & $-13.6 \pm 13.54$ & $.028 *$ & -0.398 & 0.695 & -3.91 & $.001^{*}$ \\
\hline $\begin{array}{l}\text { Maximum Knee flex. } \\
\text { Moment (N.mm/ Kg) }\end{array}$ & $27.23 \pm 6.98$ & $22.96 \pm 7.27$ & $.001^{*}$ & $29.68 \pm 11.87$ & $28.99 \pm 12.07$ & $.002^{*}$ & 0.564 & 0.579 & 2.354 & $.013^{*}$ \\
\hline $\begin{array}{l}\text { Maximum Ankle dorsiflex } \\
\text { moment (N.mm/ } \mathrm{Kg} \text { ) }\end{array}$ & $-2.60 \pm 1.05$ & $-5.69 \pm 1.87$ & $.000 *$ & $-2.88 \pm 1.52$ & $-3.49 \pm 1.83$ & $.040^{*}$ & -0.466 & 0.647 & 2.654 & $.016^{*}$ \\
\hline
\end{tabular}

Graph 1: Significant differences in knee-ankle kinetics and kinematics between groups after therapeutic interventions.

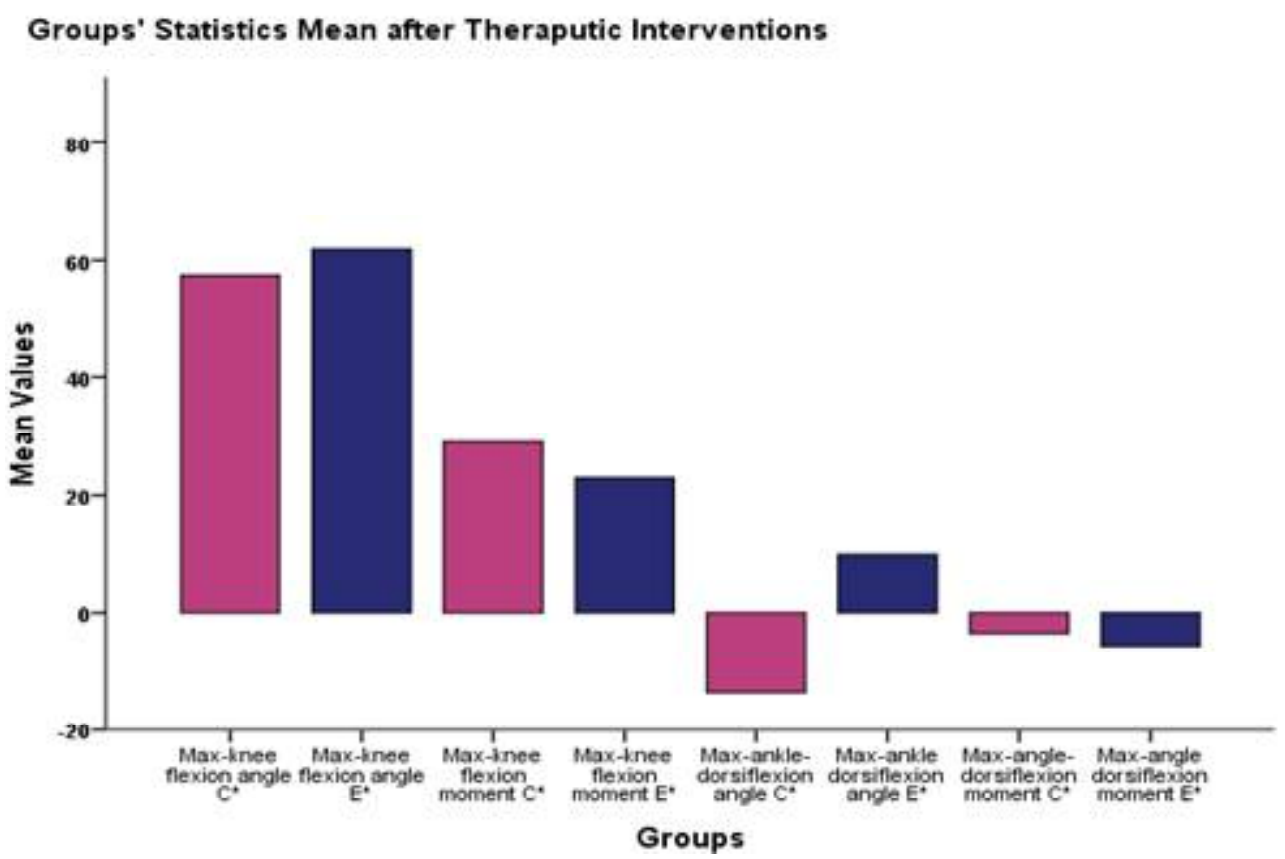


length $(p=0.001)$. Significant differences were also observed with maximum knee flexion angle, maximum ankle dorsiflexion angle, maximum knee flexion moment and maximum ankle dorsiflexion moment $(P=0.009,0.028,0.002$ and 0.040 respectively). The single limb support time showed also a nonsignificant difference between the baseline and follow up measures ( $p=0.421$ ). Within groups comparison (table 2 ) showed that there are no significant differences between all variables of the study before interventions. On the other hand, after intervention there were significant differences between cadence $(p=0.014)$, Walking speed $(p=0.037)$, Step length $(p=0.002)$, Stride length $(p=0.003)$. Significant differences were also observed with maximum knee flexion angle, maximum ankle dorsiflexion angle, maximum knee flexion moment and maximum ankle dorsiflexion moment $(P=0.004,0.001,0.013$ and 0.016 respectively) (Figure 1). The single limb support time showed also a nonsignificant difference between the baseline and follow up measures $(p=0.517)$.

\section{DISCUSSION}

The main purpose of this study was to study the effects of task oriented treadmill training on spatiotemporal, kinematic and kinetic parameters of gait in children with diplegic CP. Based on the results of gait analysis, task oriented treadmill training showed greater improvement than traditional physiotherapy program. These results also make it necessary to include such approaches in daily therapeutic programs. Our results can be attributed to treadmill training providing opportunity to repetitively train the entire gait cycle and facilitate an improved gait pattern with an increased walking speed and distance $[15,16]$. Treadmill walking exercise has been associated with increased strength and decreases in energy expenditure [17]. It also elicits stepping practice is task-specific for walking and it incorporates many of the principles of neuroplasticity [18].

The results of this study are consistent with Provost et al., who found significant improvement in velocity of walking and energy expenditure were observed and concluded that treadmill training may be an effective intervention for some ambulatory children with CP [16].
Kim and colleagues results also revealed that treadmill walking exercise improved gait efficiency in adults with CP [19]. It has been associated with decreased energy expenditure measured by oxygen cost during over ground walking. M oreover, over ground gait velocity increased post adaptation due to increased stride length and decreased stride duration after $M$ otor adaptation during treadmill walking [20]. Lee and Hidler results also suggested that when neurologically impaired individuals walk on a treadmill, they modify their muscle activation patterns and subsequently joint moments and powers [21].

In the control group, our results are supported by the results obtained by Eek and colleagues [22]. The authors found that eight weeks of individually-designed training with a focus on muscle strength not only increased muscle strength, but also improved gait function in children with CP. Increase in muscle strength is one of the major factors for the improvement in gait function so the current results support muscle strength training as a means of improving gait in children with CP. In the meta-analysis of the effect of strengthening interventions in individuals with cerebral palsy by Park and $\mathrm{Kim}$, the author concluded that strengthening exercises could increase muscle strength and improve gait for individuals with cerebral palsy, specifically for children or young people [23].

Our results also showed that there is a nonsignificant difference between single limb support time. This can be attributed to the neuromuscular disturbance which is considered as the major problem in spastic diplegic children. It leads to the disturbance of motor control and the abnormal muscle contraction patterns [24]. Spasticity causes wide range of ambulatory disorders which explains the individual difficulties in maintaining and restores both static and dynamic balance [25]. There is a strong relationship between balance and single leg support time which indicated as test to measure the upright postural stability after upper motor neuron lesion [26]. Further studies are also needed for determining more differences in the biomechanical strategies adopted by different types and severities of the cerebral palsy in order to develop better training programs. 


\section{CONCLUSION}

The result of this study revealed significant improvements in gait biomechanical parameters in swing phase and spatiotemporal parameters after using the treadmill gait training.

\section{ABBREVIATIONS}

C- Control Group

E - Experimental group

\section{ACKNOWLEDGEMENTS}

The authors wish to thank all participants for their participation and support of this project.

\section{Conflicts of interest: None}

\section{REFERENCES}

[1]. Rosenbaum P, Paneth N, Leviton A, et al. A report: the definition and classification of cerebral palsy April 2006. Dev Med Child Neurol Suppl. 2007;109:8-14.

[2]. Christensen D, Van Naarden Braun K, Doernberg NS, et al. Prevalence of cerebral palsy, co-occurring autism spectrum disorders, and motor functioning. Autism and Developmental Disabilities M onitoring Network, USA, 2008. Dev Med Child Neurol. 2014; 56(1):59-65.

[3]. Boyle CA, Boulet S, Schieve LA, et al. Trends in the prevalence of developmental disabilities in US children, 1997-2008. Pediatrics. 2011;127(6):10341042.

[4]. Al Salloum AA, El M ouzan M I, Al Omar AA, Herbish AS, Qurashi MM. The prevalence of neurological disorders in Saudi children: a community-based study. J Child Neurol. 2011;26(1):21-24.

[5]. Aye T, Thein S, Hlaing T. Effects of strength training program on hip extensors and knee extensors strength of lower limb in children with spastic diplegic cerebral palsy. J Phys Ther Sci. 2016; 28(2) :671-676.

[6]. Fowler EG, Goldberg EJ. The effect of lower extremity selective voluntary motor control on interjoint coordination during gait in children with spastic diplegic cerebral palsy. Gait Posture. 2009;29(1):102-107.

[7]. de M oraisFilho M C, Kawamura CM, LopesJA, Neves DL, Cardoso M de O, Caiafa JB. Most frequent gait patterns in diplegic. Acta Ortop Bras. 2014; 22(4): 197-201.

[8]. Rodda JM , Graham HK, Carson L, Galea MP, Wolfe R. Sagittal gait patterns in spastic diplegia.J Bone Joint Surg Br. 2004;86(2):251-258.

[9]. Shumway-Cook, A, and Woollacott, M H: Motor Control: Translating Research into Clinical Practice, ed 4. Lippincott Williams\& Wilkins, Philadelphia, 2012.

[10]. LerouxA, Pinet H, Nadeau S. Task-oriented intervention in chronic stroke: changes in clinical and labo ratory measures of balance and mobility. Am J Phys Med Rehabil, 2006;85:820-830.

[11]. Salbach NM, M ayo NE, Wood-Dauphinee S, Hanley JA, Richards CL, Côté R. A task-orientated intervention enhances walking distance and speed in the first year post stroke:a randomized controlled trial. Clin Rehabil, 2004;18:509-519.

[12]. Kim Y, Lee BH. Clinical Usefulness of Child-centered Task-oriented Training on Balance Ability in Cerebral Palsy. J Phys Ther Sci. 2013;25(8):947-951.

[13]. Su IY, Chung KK, Chow DH. Treadmill training with partial body weight support compared with conventional gait training for low-functioning children and adolescents with nonspastic cerebral palsy: a two-period crossover study. Prosthet Orthot Int. 2013;37(6):445-453.

[14]. Grecco LAC, Tomita SM, Pasini H, Sampaio LM, Oliveira CS. Effect of treadmill gait training on static and functional balance in children with cerebral palsy: a randomized controlled trial. 2013;17(1):1723.

[15]. Willoughby KL, Dodd KJ, Shields N, Foley S. Efficacy of partial body weight-supported treadmill training compared with overground walking practice for children with cerebral palsy: a randomized controlled trial. Arch Phys Med Rehabil 2010;91:333339.

[16]. Provost B, Dieruf K, Burtner PA, PhillipsJP, BernitskyBeddingfield A, Sullivan KJ, et al. Endurance and gait in children with cerebral palsy after intensive body weight-supported treadmill training. Pediatr Phys Ther 2007;19:2-10.

[17]. Dodd KJ, Foley S. Partial body-weight-supported treadmill training can improve walking in children with cerebral palsy: a clinical controlled trial. Dev Med Child Neurol 2007;49:101-105.

[18]. Kleim JA, Jones TA. Principles of experience-dependent neural plasticity: implications for rehabilitation after brain damage. J Speech Lang Hear Res. 2008;51:S225-S239.

[19]. Kim OY, Shin YK, Yoon YK, Ko EJ, Cho SR. The effect of treadmill exercise on gait efficiency during overground walking in adults with cerebral palsy. Ann Rehabil Med. 2015;39(1):25-31.

[20]. Savin DN, M orton SM, Whitall J. Generalization of improved step length symmetry from treadmill to over ground walking in persons with stroke and hemiparesis. Clin Neurophysiol. 2014;125(5):10121020.

[21]. Lee S], Hidler J. Biomechanics of overground vs. treadmill walking in healthy individuals. J Appl Physiol. 2008;104(3):747-755.

[22]. Eek M N, Tranberg R, Zügner R, Alkema K, BeckungE. Muscle strength training to improve gait function in children with cerebral palsy. Dev Med Child Neurol. 2008;50(10):759-764.

[23]. Park EY, Kim WH. Meta-analysis of the effect of strengthening interventions in individuals with cerebral palsy. Research in Developmental Disabilities 2014;35(2):239-249. 
[24]. Emara H. Effect of a new physical therapy concept on dynamic balance in children with spastic diplegic cerebral palsy. EJMHG 2015;16(1):77-83.

[25]. Kazon S, Grecco LA, Pasini H, Corrêa JC, Christovão TC, de CarvalhoPde T, et al. Static balance and function in children with cerebral palsy submitted to neuromuscular block and neuromuscular electrical stimulation: study protocol for prospective, randomized, controlled trial. BM C Pediatr. 2012; 16:12:53.
[26]. Johnston TE, Watson KE, Ross SA, Gates PE, Gaughan JP, Lauer RT, Tucker CA, EngsbergJR. Effects of a supported speed treadmill training exercise program on impairment and function for children with cerebral palsy. Dev Med Child Neurol. 2011;53(8):742750.

\footnotetext{
How to cite this article:

$M$ ariam A. Ameer, M ohamed E. Khallaf. EFFECT OF TREADM ILL GAIT TRAINING ON DIPLEGIC CHILDREN SWING PHASE GAIT PARAM ETERS. Int J Physiother Res 2017;5(1):1829-1835. DOI: 10.16965/ijpr.2016.200
} 